\title{
EMBRIO v2.0 - A Virtual Learning Environment for Embryology Teaching
}

\author{
Leandro Guimarães Garcia, Mexwendell Gomes de Moraes, Gustavo Macedo Rodrigues, and Dábila \\ Cristina dos Santos
}

\begin{abstract}
This work presents a virtual learning environment that contains three-dimensional models of human embryos and fetuses. They are based in extensive bibliographic research, which makes them totally interactive learning objects. These models were generated in an open source software called BLENDER, and constitute a similar representation of human embryos and fetuses.
\end{abstract}

Index Terms-Embryology, learning objects, learning health system, virtual learning environment, BLENDER.

\section{INTRODUCTION}

Most of human embryology books treat the formation of the body systems in separated chapters. This approach generates a fragmented knowledge, disconnecting temporarily the state of development of a body system in relation to another.

This knowledge fragmentation represents a problem during clinical treatment because, when a pregnant woman looks for a health professional, she is in a specific phase of gestation, and all the conceptus' systems are in a certain degree of maturation. Thereby, the professional is required to know de approximate state of maturation of most of the conceptus' organs, in the specific gestational phase of the patient when she is treated. However, how can we demand a holistic knowledge from a professional who has learnt about the development of the body systems during gestational period in a fragmented way at the subject of Embryology?

To address these questions, our suggestion is that Embryology teaching should not be guided by the separated study of the body systems. Instead, Embryology teaching should be guided with emphasis to the development period, where all the body systems could be analyzed simultaneously according to maturation phase. This approach carries the promise of solving the problem of knowledge fragmentation of this area.

To accomplish this goal, our research group is working on Virtual Learning Environment (VLE) [1] called EMBRIO [2] This framework has many different learning objects, which

Manuscript received May 1, 2015; revised January 15, 2016. This work was supported in part by the CNPq and UFT for some schoolarships to a portion of the students who helped on this work.

L. G. Garcia is with the Mestrado em Modelagem Computacional de Sistemas and School of Medicine, Universidade Federal do Tocantins, Av. NS 15, 109 N, Palmas, TO 77001-090, Brazil (e-mail: lggarcia@uft.edu.br)

M. G. de Moraes, G. M. Rodrigues, and D. C. dos Santos are with the Faculdade de Ciência da Computação, Universidade Federal do Tocantins, Av. NS 15, 109 N, Palmas, TO 77001-090, Brazil (e-mail: mexwendell@mail.uft.edu.br, dabilacris@hotmail.com). are actually three-dimensional interactive models of human embryos and fetuses in periods where changes in body systems are faster and hard to understand. The VLE EMBRIO allows the student to evaluate all the three-dimensional (3D) structure of the conceptus in a certain period of gestation. Hence, the student may evaluate the maturation degree of all body systems, simultaneously, approaching Embryology teaching to the reality of daily clinic.

Despite of many similar initiatives [3]-[7], this work is differs from them because it is based exclusively in published scientific material [8]-[13] instead of real bodies or images of embryos or fetuses obtained by Magnetic Resonance Imaging.

In this paper we present the second version of this system (EMBRIO v2.0), in which both the user's graphic interface of the VLE outline and the learning objects (3D interactive models of human embryos and fetuses modeled on BLENDER) were extensively refined. The three-dimensional models of the conceptus that already existed on the first version of VLE were reviewed using a bibliography that differs from the one used to generate them [14], [15]. In this process, all three-dimensional models in the first version of VLE were extensively discussed based on the review bibliography and adjusted after a consensus within the research group. The user's graphic interface of VLE was also discussed and adjusted to be more intuitive. At the end, a new model was implemented to the VLE, the one correspondent to the 12-week-old conceptus.

The decision to add a three-dimensional model of a 12-week-old conceptus was taken by didactic reasons after an agreement within the research group. By the age of 12 weeks, the conceptus presents a mature cardiovascular system, in relation to the 8-weeks-old one. Besides, the intestine herniation that existed at 8 weeks doesn't exist at 12 weeks. Finally, the urogenital system is already developed and differentiated, which didn't happen at the 8th week. For these reasons, we considered that, to an improved learning of the fast changes that occur in embryology would be essential to add a three-dimensional model of the 12-week-old conceptus to the VLE. We also believe that it will not be necessary to add older models, because all morphological changes that happen after this time may be easily understood by the students.

\section{SYSTEM'S DESCRIPTION}

VLE EMBRIO v2.0 starts with the name of the institution where it was created and a button with the name of the system (Fig. 1). When the student clicks this button, there are two 
options: studying three-dimensional models of human embryos or fetuses or ultrasonography videos (Fig. 2).

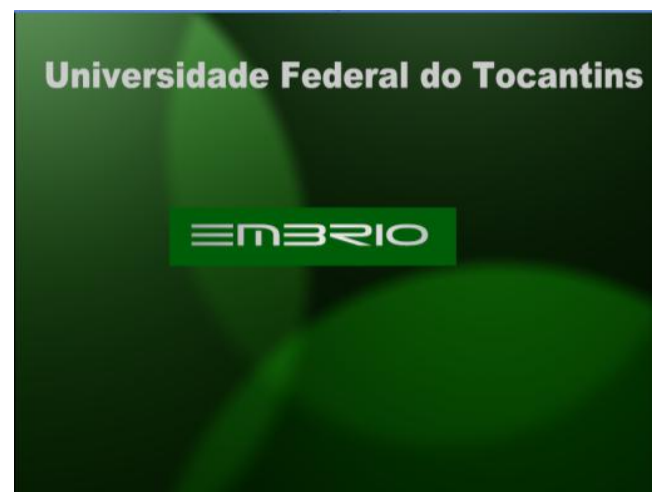

Fig. 1. First screen of the system menu EMBRIO v2.0.

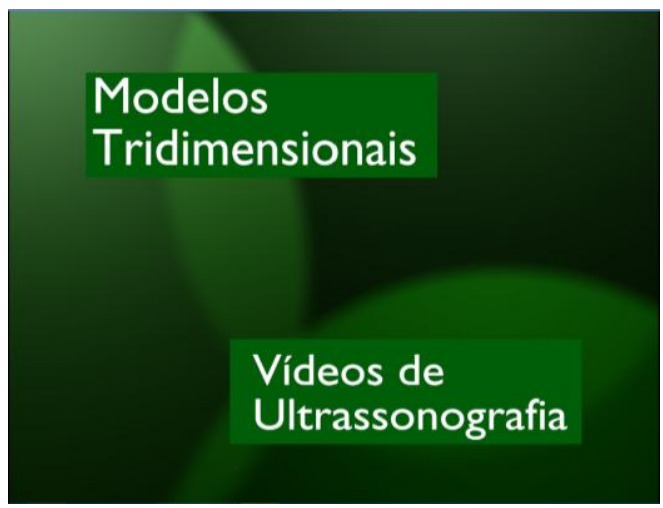

Fig. 2. Second screen of the system menu EMBRIO v2.0.

In the case of our VLE, there aren't ultrasonography videos available yet. When clicking at the button to visualize the 3D-models of human embryos or fetuses we can see several options according to the age of the conceptus (Fig. 3). The user can choose any age present in this menu, and to illustrate the system, we chose the button Terceira Semana (Third Week) on Fig. 3, which leads to Fig. 4.

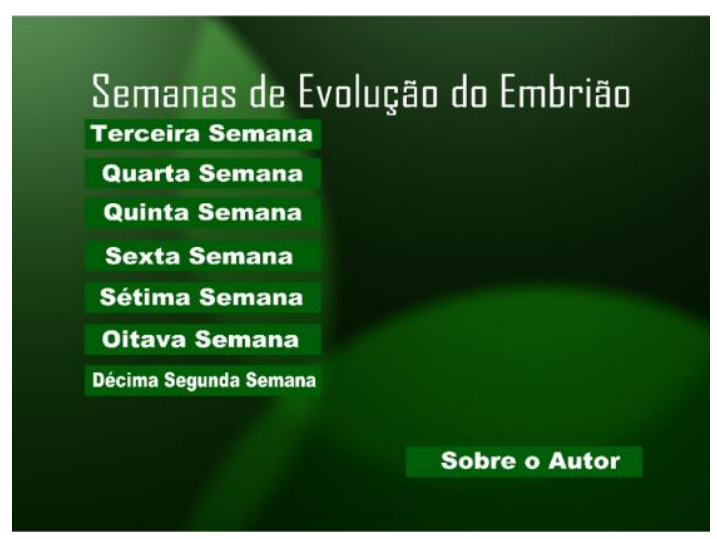

Fig. 3. Third screen of the system menu EMBRIO v2.0.

The user can choose any of the periods observed on Fig. 4. To demonstrate, we chose to watch and study the model relative to 21 days of embryonic life (Fig. 5)

We can see many features that the graphical interface offer to the user, numbered from 1 to 9 , to ease the use of the system. Feature 1 transports the user to the screen observed on Fig. 3. Feature 2 cuts the conceptus transversely (white circle) or longitudinally (blue circle). It's important to highlight that for all ages within the third week it's possible to visualize transversal and longitudinal cuts of the conceptus for better understanding of the 3D-model. Features associated to number 3 allow the user to move the 3D-model using the mouse (there are also keyboard shortcuts to do the same thing). Number 4 allows to return to the complete model from its cut. Feature 5 turns off the system. Features associated to number 6 allow to navigate the system, going to the previous 3D-models (left arrow) or next models (right arrow). Number 7 represents subtitles that show the names of the conceptus' parts when we pass the mouse over them. Feature number 8 represents a menu that allows removing parts of the conceptus to study the relationship among its internal structures. Number 9 removes the frame containing all features and leaves only the conceptus in the center of the screen.

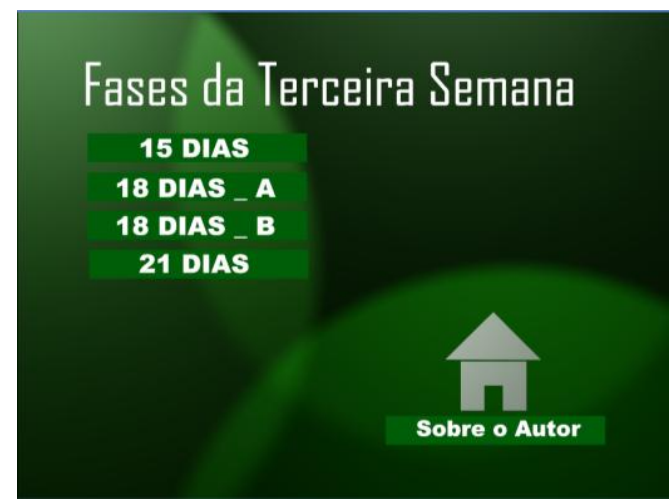

Fig. 4. Screen related to the choice of the option Terceira Semana (Third week), from the system menu EMBRIO v2.0.

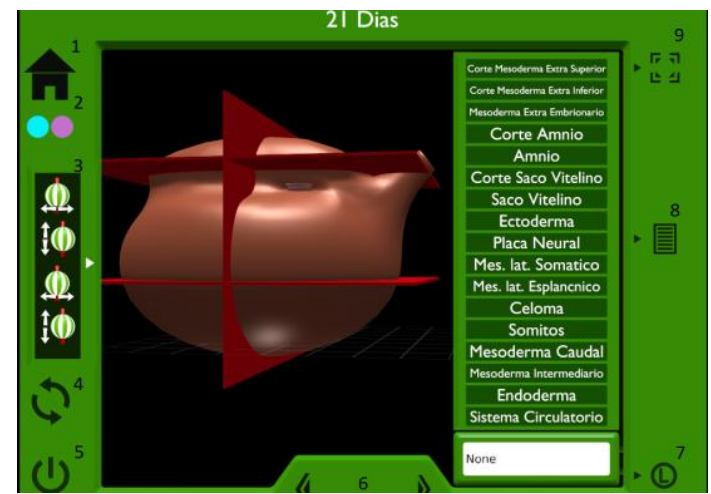

Fig. 5. Three-dimensional schematic model of a 21-day embryo where features of graphical interface are numbered from 1 to 9 .

Fig. 6 shows a transversal cut of the 21-day conceptus from Fig. 5. This model is generated after clicking in the white circle associated to number 2 .

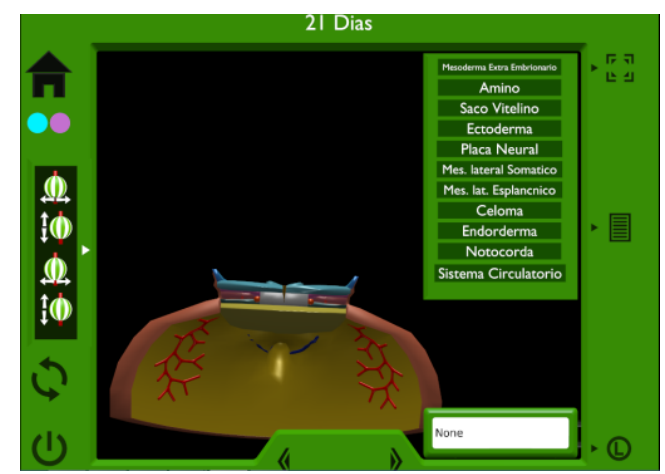

Fig. 6. Transversal cut from the 21-day conceptus observed on Fig. 5.

The VLE presents four three-dimensional models of conceptus in third week (related to day 15, beginning of day 18 , final of day 18 and day 21), four three-dimensional 
models of conceptus in fourth week (relative to days 22, 24,26 and 28) and only one 3D-dimensional model of conceptus in fifth, sixth, seventh, eighth, and twelfth week, respectively.

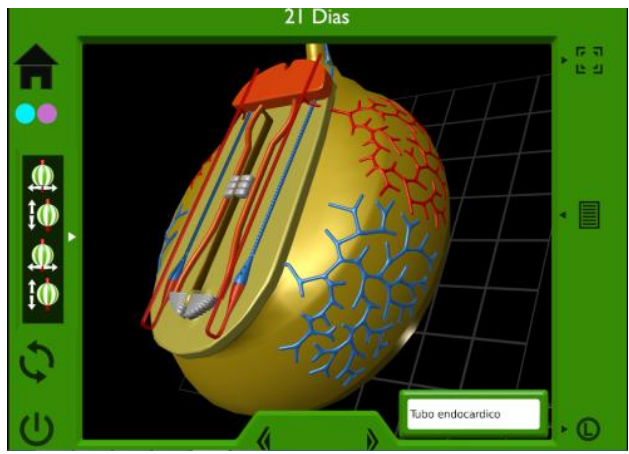

(a)
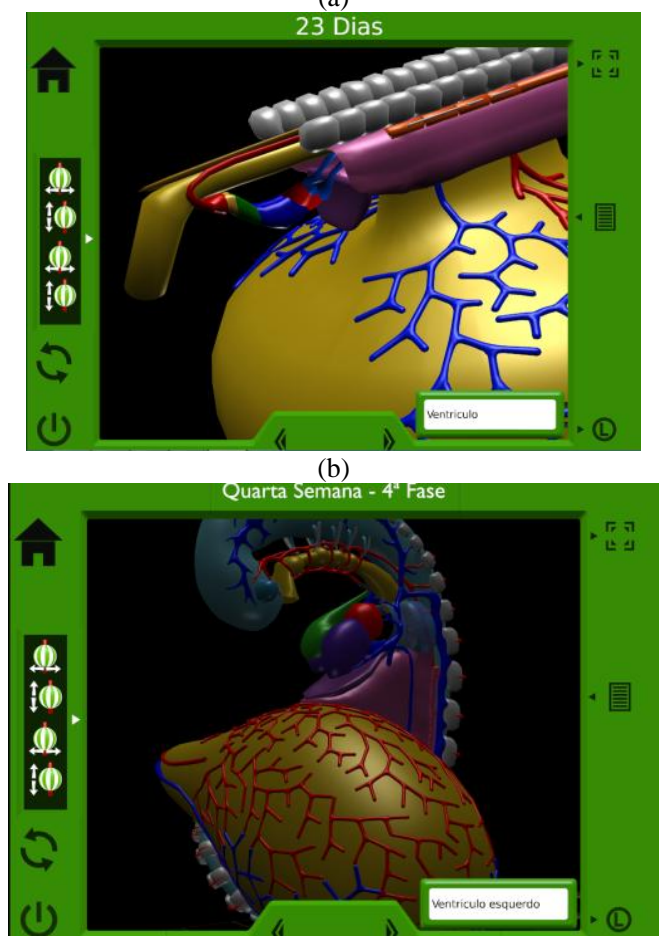

(c)

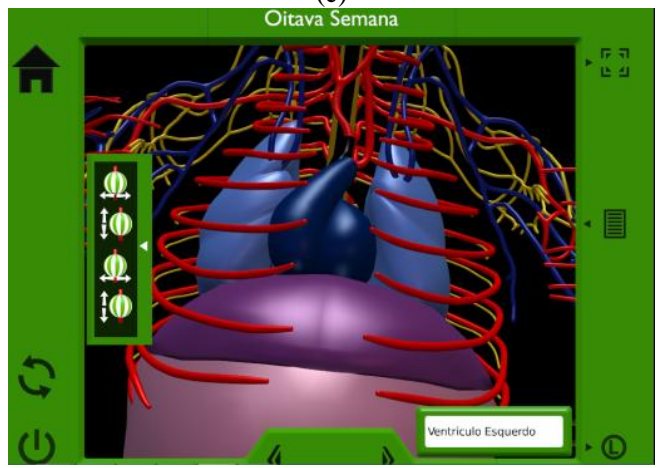

(d)

Fig. 6. Many phases of the heart development during the embryonic period. In A the embryo is 21 days old and the heart is represented by two separated endocardial tubes. In B the embryo is 22 days old and the endocardial tubes are merged. In $\mathrm{C}$ the embryo is 28 days old and the heart has a cubic shape. In $\mathrm{D}$, by the age of 8 weeks, the heart presents a format similar to its definitive shape.

From this sequence of 3D-models of conceptus of human embryos or fetuses it is easy for the student to understand complex changes in the study of morphogenis in embryology. The transformations in the shape of the structures studied before just in two-dimensional level in books, from pictures, can now be visualized in three dimensions in a completely interactive way. Besides, relations among the structures that are hard to understand get much easier to be visualized in three-dimensional models. Fig. 7 and Fig. 8 clearly show how this is possible with the use of this VLE.
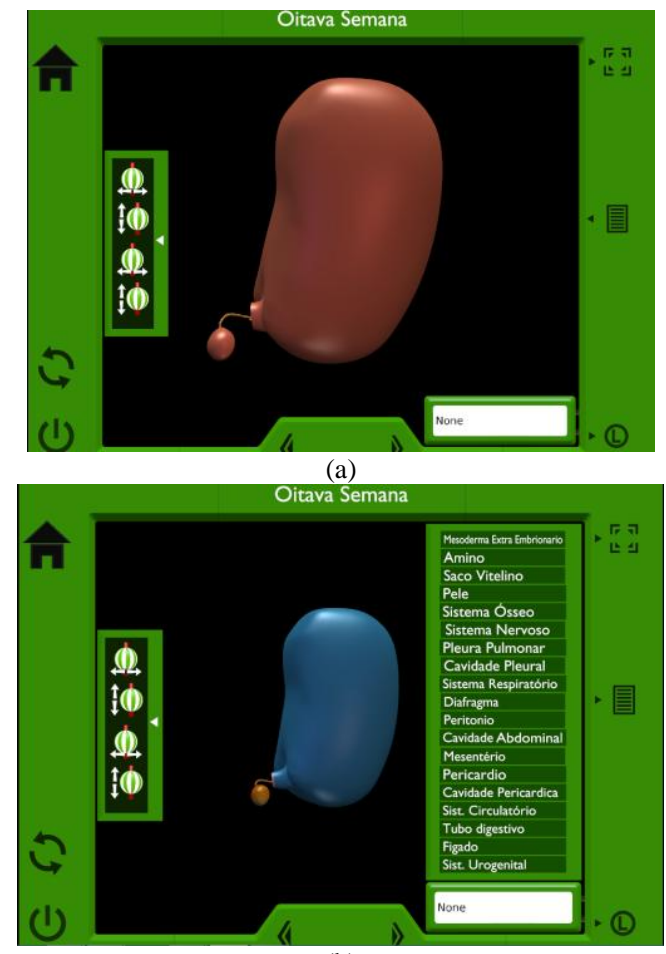

(b)

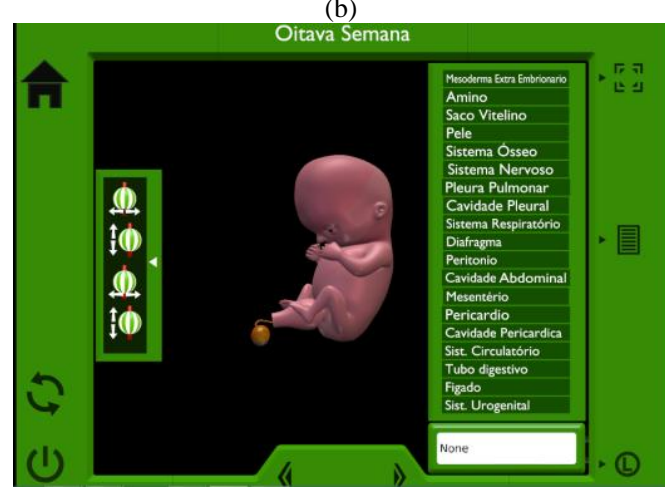

(c)

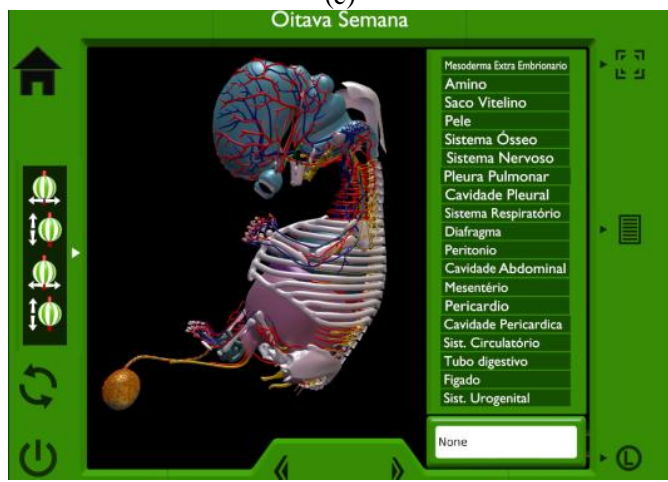

(d)

Fig. 8. 8-weeks-old conceptus seen inwardly. In A we can see just the extra embryonic mesoderm. In B we can see the amnio that stays under the extra embryonic mesoderm. In $\mathrm{C}$ we can see the skin under the amnio. In D we see the structures under the skin.

\section{CONCLUSION}

From the arguments presented we concluded that it's possible to generate pictorial models of embryos and fetuses from scientific material extracted from many research sources. 
We have also verified that it is possible to group these virtual objects in a rational and sequential way with the objective to generate holistic and temporally connected knowledge about embryonic and fetal development. Our next step will be a further analysis of the impact of the use of this VLE in Embryology teaching in health courses, upward the insertion of ultrasonography videos in several different moments of the gestational period.

Up to the authors knowledge, there exist not a similar system to EMBRIO 2.0 and that characterizes its innovation. The other systems that allow observing and turning the conceptus three-dimensionally that are being developed by other research groups present just a few three-dimensional models of embryos, usually related only do the embryonic period. Our VLE, on the other hand, brings around 20 three-dimensional models (considering the cuts in the conceptus in the third week of embryonic development), in many different gestational ages, covering the embryonic period and part of the fetal period.

Additionally, another innovation is that the 3D-models presented were generated by a graphical modeling program, using many bibliographic sources, while other systems developed by other research groups were created, in general, from images of magnetic resonance imaging (MRI). This type of imagery overlaps close structures and presents a very different appearance from the ones present in text books and that increases the difficulty of students to understand embryology.

\section{ACKNOWLEDGMENT}

We thanks CNPq and UFT for PIBIC schoolarships for some students who participated on this work.

\section{REFERENCES}

[1] M. Weller, Virtual Learning Environments: Using, Choosing Developing Your VLE, 1st ed., Routledge, 2007.

[2] L. G. Garcia, D. A. Pacheco, R. L. Sousa, R. S. Santos, and F. H. R. Brune, "EMBRIO - A 3D software for embryology learning," International Proceedings of Economics Development and Research, pp. 73-78, vol. 41, 2012.

[3] K. Kakusho, S. Mizuta, Y. Minekura, M. Minoh, T. Nakatsu, and K. Shiota, "Illustrating human development by computer graphics for education in embryology," in Proc. the International Conference on Computers in Education, 2001, pp. 412-415.

[4] S. Mizuta, K. Kakusho, Y. Minekura, M. Minoh, T. Nakatsu, and K. Shiota, "Construction and application of 3D model sequence to illustrate the development of human embryo," in Proc. SPIE /Medical Imaging, vol. 4681, 2002, pp. 732-741.

[5] R. Abdulla, G. A. Blew, and M. J. Holterman, "Cardiovascular embryology," Pediatric Cardiology, pp. 191-200, vol. 25, 2004.

[6] S. Yamada, C. Uwabe, T. Nakatsu-Komatsu et al., "Graphic and movie illustrations of human prenatal development and their application to embryological education based on the human embryo specimens in the
Kyoto collection," Developmental Dynamics, pp. 468-477, vol. 235, 2006.

[7] J.J. Azkue, "A digital tool for three-dimensional visualization and annotation in Anatomy and Embryology learning," European Journal of Anatomy, pp. 146-154, vol. 17, 2013.

[8] J. Hib, Embriologia Médica, 1 st ed., Guanabara Koogan, 2007.

[9] K. L. Moore and T. V. N. Persaud, Embriologia Clínica, 8th ed., Elsevier, 2008

[10] T. W. Sadler, Langman's Medical Embryology, 12th ed., Lippincott Wiliams \& Wilkins, 2011.

[11] G. C. Schoenwolf et al., Larsen Embriologia Humana, 4th ed., Elsevier, 2009

[12] J. W. Rohen, Embriologia Funcional, 2nd ed., Guanabara Koogan, 2005.

[13] G. Rager et al., Human Embryology and Teratology - A Concise Course, 3rd ed., Embryo CD, 2008.

[14] C. R. Bardeen et al., Manual of Human Embryology, 1st ed., J. B. Lippincott, 1912.

[15] L. R. Cochard, Atlas de Embriologia Humana de Netter, 1st ed., Elsevier, 2014

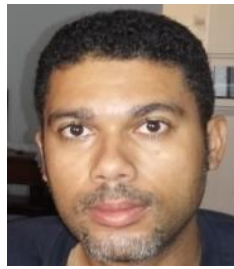

Leandro Guimarães Garcia was born in the state of Goiás, Brazil in 1978. He has a bachelor's degree in biomedicine from the Federal University of São Paulo, Brazil and finished his doctorate in cell and molecular biology in the University of Brasilia, Brazil in 2006.

He worked as teacher and coordinator of the biomedicine undergraduate course of the Faculty of Higher Education of the United Amazon (FESAR) and currently is an associate professor at the Federal University of Tocantins. In this university he works developing ELVs for embryology, parasitology, and human anatomy teaching. Currently he also works with research and development in the field of health computing.

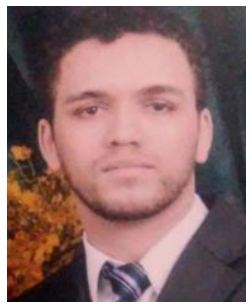

Mexwendell G. de Moraes was born in the state of Maranhão, Brazil in 1991. He is an undergraduate student of computer science at the Federal University of Tocantins, Brazil. He has experience with $3 \mathrm{D}$ modeling and animation and with game programming. He participated in the $3 \mathrm{D}$ animated short film. He also contributed with the short film, "Praça dos Girassóis", sponsored by the Municipal Cultural Incentive Program (Promic) of 2013.

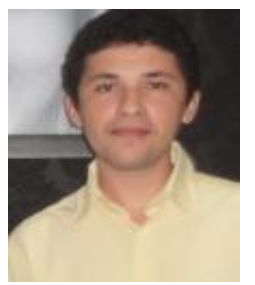

Gustavo Macedo Rodrigues was born in the state of Tocantins, Brazil in 1992. He is an undergraduate student of computer science at the Federal University of Tocantins, Brazil. It has experience with 3D modeling and programming.

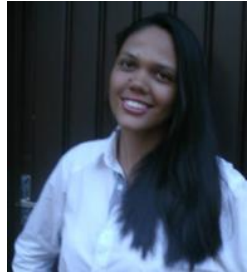

Dábila Cristina dos Santos was born in the state of Goiás, Brazil in 1992. She holds a bachelor of computer science from the Federal University of Tocantins, Brazil, in 2015. She worked with 3D modeling, but currently works at the programming lab of the Federal University of Tocantins. 